background of inflammation. The histogenesis of the tumour was not clear although the differentials of melanoma, olfactory neuroblastoma, neuroendocrine tumour and undifferentiated carcinoma were considered most likely. Fontana Masson stain was performed to detect melanin granules and was weakly positive. Immunohistochemistry demonstrated strong staining of the neoplastic cells for PNL-2 and MelanA. Eight percent of the cells stained positively for cytokeratin and $20 \%$ for vimentin. These results were considered diagnostic of a melanoma.

The dog received a hypofractionated radiotherapy protocol (four, weekly fractions of 8 Gray) and achieved a complete clinical response which persists at the time of writing (4 months). To the author's knowledge, this is the first report of the diagnosis and successful treatment of a melanoma in the nasal cavity of a dog.

\section{Approaching tumour heterogeneity using laser capture microdissection coupled with gene expression on formalin-fixed paraffin canine mammary tumours}

\section{Silvia Guil-Luna', Francisco Javier Salguero'2, Jaime Gómez-Laguna', Abdullah Alijojani ${ }^{2}$, Waldo García- Jimenez ${ }^{2}$, Silvana Di Palma ${ }^{2}$, Yolanda Millán', Raquel Sánchez-Céspedes', Juana Martín de las Mulas ${ }^{1}$}

\footnotetext{
1 Department of Comparative Pathology, University of Córdoba, Córdoba, Spain

2 Department of Pathology and Infectioous Diseases, School of Veterinary Medicine, Surrey, UK
}

Canine mammary tumours (CMT), as its human counterpart, are characterized by cellular heterogeneity. The concept of tumour heterogeneity is increasingly being recognised since it will be important for understanding the molecular pathogenesis of these tumours. The use of laser capture microdissection (LCMD) in formalin-fixed, paraffin-embedded (FFPE) tissues is a recent methodology in pathology that permits the rapid and reliable extraction of RNA from different pure cell populations. The combination of LCMD and gene analysis has the potential to reveal novel pathogenic pathways and biomarkers by which normal cells progress to invasive malignant cells. However, in order to get high-quality RNA from FFPE, optimization of protocols has to be previously performed. The goals of this study were first, to validate the LCMD technique in CMT by using two different slide types (glass membrane and frame membrane); second, to validate two commercial kits for RNA extraction; and third, to identify selected tumour biomarkers of interest in obtained samples. Thirty-nine FFPE samples from benign and malignant CMT were selected to identify normal epithelial and myoepithelial cells, malignant epithelial and myoepithelial cells and stroma cells by Arcturus $^{\circledR}$ LCMD. RNA extraction was done by the RecoverAll Total Nucleic Acid Isolation Optimized for FFPE samples (Ambion) and by RNAqueous Micro-kit (Ambion). Gene expression by using classic polymerase chain reaction (PCR) was performed in order to study the expression of progesterone receptor (PR) and the antiangiogenic markers VEGFA and VEGFR2. HPTR1 and RPL32 were used as housekeeping genes. Isolation of the different cell populations was successfully performed by using PEN-Membrane Glass slides. Whereas RecoverAll Total Nucleic Acid Isolation Optimized for FFPE samples yielded poor RNA concentration and quality by NanoDrop, RNAqueous Micro-kit produced a significant improvement in the RNA concentration and quality and allowed the success amplification for both housekeeping and tumour biomarkers genes. In conclusion, LCMD coupled with RNA extraction by the RNAqueous Micro-kit and classic PCR is a reliable methodology for the molecular characterization of these heterogeneous cell populations in FFPE samples of CMT. However, further studies are required to optimize this methodology in order to get a better understanding of tumour heterogeneity.

\section{Retrospective evaluation of} metronomic cyclophosphamide in epithelial and mesenchymal malignant tumours

\section{J Ellis, RD Foale}

Dick White Referrals, Six Mile Bottom, Cambridgeshire, UK

Continuous low dose chemotherapy administration has been reported to have anti-angiogenic effects with efficacy against multiple tumour types. This study aimed to retrospectively assess the use of metronomic cyclophosphamide for histopathologically confirmed solid malignant canine tumours.

Patient records from 2009 to 2014 were reviewed at a multi-disciplinary referral hospital. Dogs without histological confirmation of diagnosis were excluded. Thirty-seven cases of epithelial or mesenchymal malignancy in dogs treated with $10 \mathrm{mg}$ cyclophosphamide daily/every other day were identified. This included 14 high grade soft tissue sarcomas, 5 soft tissue sarcomas of undefined grade, 4 intermediate grade soft tissue sarcomas, 3 splenic haemangiosarcomas, 3 incompletely excised low grade soft tissue sarcomas, 2 thyroid carcinomas, and 1 each of adenocarcinoma, bladder haemangiosarcoma, splenic fibrosarcoma, apocrine gland carcinoma, metastatic prostate carcinoma, and metastatic carcinoma of unknown origin. Two were lost to follow up. Twenty-two had at least one attempt at surgical excision and three underwent radiotherapy prior to initiation of chemotherapy.

Three of the dogs (8.6\%) developed haematuria, but no other adverse effects were reported. The haematuria resolved in all three dogs after cessation of cyclophosphamide. 